\title{
Comment
}

DO WE KNOW THE VALUE OF WHAT WE ARE DOING?

\section{Evolution of evaluation in science museums and centres}

\section{Carmen Sanchez-Mora}

\begin{abstract}
The great increase in visitor studies on science museums and centres (SMC) has been marked also by a shift in approach to these studies, paying more attention to the social context of the visits and the nature of the experience. Evaluations have influenced directly SMC exhibition practices but more attention needs to be paid in research about the personal experiences of visits, how these are interpreted and how they contribute to scientific literacy.
\end{abstract}

Their proliferation, their ability to reach adults long after schooling, the possibility of bringing children closer to science after the known failures of formal education - all these could be reasons to support the view (especially in the face of their detractors) that science museums and centres (SMC) are important media for social communication of science.

This seems to be clear to the institutions themselves, which often state their mission in these terms: to communicate science, to bring it closer to the public and to increase awareness of it; and the one that seems to be most ambitious: to make science part of culture.

Over 30 years ago we thought that SMC were accomplishing that mission, but soon we began to worry about how and what we were communicating about science, two questions that could only be answered by evaluation (to solve particular problems or justify ourselves to our sponsors), or by research (to produce new knowledge). In both processes we usually use the same techniques, such as surveys, questionnaires, tracking of visitors, observations, etc.; the difference between the two depends on the goals of our task.

In both cases, the data are obtained from visitors, whether we seek to evaluate their experience in the SMC, or to evaluate the exhibits themselves. So, in the 1970s and 1980s we began to work with experimental designs that had to define $a$ priori the possible results of the experience, consequently leaving out many important results. After that, we moved from behavioural to cognitive studies, and subsequently to socio-cognitive ones, giving increasing importance to the social context of the SMC experience.

Among the many examples of these various approaches, we can mention Diamond (1986) who studied family behaviour on museum visits, Borun et al. (1993) who investigated the naive conceptions visitors bring to an exhibit and the consequent knowledge 
gain, and McManus (1989) who analysed the social discourse at different exhibits at the National History Museum in London and demonstrated that the style of exhibit deeply affects the kind of thinking visitors engage in [1-3].

As a result of more recent approaches in evaluation, we learned to improve our displays and to control some of the design features of the exhibits and the context of the visit. We knew at that time that learning could be shaped, and that if we controlled the exhibits' power of attraction, the content of the labels beside displays, the distribution of these in the exhibition space, the pre and post-visit activities, and the purpose of the visit, among others, this could lead us to different forms of physical and dialogical exchange with the displayed themes, and, as a result, different degrees of attention, encoding and recall of learned scientific concepts would emerge.

However, we were still tied to a vision of the experience in the SMC based on the acquisition of concepts; there were few examples at that time of exhibits or of visitor studies giving importance to non-cognitive aspects of the visit, such as emotions, for example.

Slowly we realised that SMC learning occurs as a very particular phenomenon: that of informal learning, which is mainly personal and contextual and is also time-consuming. All this caused a big turn in evaluation over the last two decades: more holistic methods of evaluation were required that addressed the complexity of the process. Already in 1999 D.J. Johnston developed in his Ph.D. Thesis (Curtin University, Australia) instruments to measure short- and long-term impacts of museum visits based on written responses of visitors to a questionnaire, permitting him to use the words and phrases chosen by visitors.

Interest has grown in trying to detect the impact of brief individual learning experiences over time. Although there is not yet a suitable methodology to achieve this, the memory of the event seems to offer a way to document the learning process occurring in the SMC. However, we still face difficulties in tracking visitors after the event occurs.

The main question, I believe, for evaluation and future research, is related to our institutional mission as generators or enhancers of scientific literacy in society. Since science is the object of our exhibitions, we need to pause and think about the nature of science we display. Is the desire to let visitors have fun trivialising or even denying science, which is presented in a superficial, uncritical, unproblematic way, using simplified definitions? Are the ethical dimensions and the social components of science and technology being neglected?

Today we know that, if we expect cognitive understanding as a result of science communication, we will be disappointed. The only thing we can hope for is that after visiting the SMC we will make sense of the world in a scientific way; it does not mean that we are looking for a deep understanding of concepts soon forgotten, but only its indirect effect on many areas of our life. In other words, evaluation must be redirected to detect if we have communicated "a scientific sense of the world".

We understand that the visitor is an active participant in the construction of new knowledge and understanding, and we speak of a process rather than an outcome, so our evaluation should be inclined to use less behavioural methods and more interpretative ones. We know very little about everyday experiences related with the natural world in non-school 
contexts, and we are not even sure of the mental status of scientific intuitive concepts as a result of daily experiences.

We are having hard times, as no large-scale studies seem to show that SMC increase the public understanding of science - but then we may think that we are not evaluating correctly. At the same time we find with many contextual studies that their results can hardly be generalised. We need methods to address the whole complexity of the process, considering multiple variables, without losing validity in relation to this multidimensional reality.

Now we do not care any more if people learn science from a visit to a SMC, but we seek to know what are the relationships between the museum experience and long-term memories. It is clear, then, that we require holistic and qualitative research approaches that enable us to see how society exposed to the SMC, solves problems, understands news in the media, look to science as a human enterprise, handles certain vocabulary, makes informed decisions [4].

A new methodological path can be glimpsed in the study of cultural practices related to informal science learning; this will imply the use of methods other than those used hitherto, or maybe a shift in the conceptualisation of the SMC. Are we going to wait another 30 years in order to accomplish it?

\section{References}

[1] J. Diamond (1986), “The behaviour of family groups in science museums”, Curator 29: 139-154.

[2] M. Borun, C. Massey and T. Lutter (1993), "Naive knowledge and the design of science centre museums exhibits", Curator 36: 201-219.

[3] P. McManus (1989), “The visitor experience”, in D.L. Uzzell ed., Heritage Interpretation 2: 156-165.

[4] See, for example, L.J. Rennie and G.F. Williams (2002), "Science centres and scientific literacy: Promoting a relationship with science”, Science Education, 86: 706-726.

\section{Author}

Maria Carmen Sanchez-Mora (Master, Stanford University; Ph.D., Universidad Nacional Autónoma de México, UNAM) has for ten years been researching science communication mainly in science centres. She is co-ordinator of the science communication training group at UNAM. E-mail: masanche@universum.unam.mx.

How TO CITE: M.C. Sanchez-Mora, Evolution of evaluation in science museums and centres, JCOM 13(01)(2014)C02. 